\title{
“Electrodeless" Plasma Cathode for Neutralization of Ion Thrusters
}

\author{
Ben W. Longmier ${ }^{*}$ and Noah Hershkowitz ${ }^{\dagger}$ \\ University of Wisconsin - Madison, Madison, WI 53706
}

\begin{abstract}
Significant progress has been made in the characterization of the necessary parameters required to create an effective Radio Frequency (RF) plasma based electron source. Such a device has the promise of comparable current densities to and longer operational lifetimes than hollow cathodes. The operational lifetime of hollow cathodes is ultimately limited by cathode deterioration. RF sources provide an alternative approach that does not consume electrode material while providing electrons. Preliminary experiments suggest that an electron current of 3.75 Amps was extracted through an electron sheath near a grounded ring within an argon plasma using $340 \mathrm{~W}$ of $\mathrm{RF}$ power. Permanent magnets provided an axial magnet field of 150 Gauss at the antenna. Experimental evidence from the RF plasma cathode suggests that the amount of neutralizing electron current that can be extracted is equal to the random electron flux and is limited by the plasma density, the neutral argon flow rate, and the ion loss area provided in the source.
\end{abstract}

* Graduate Student, Engineering Physics Department, 1500 Engineering Dr., Madison WI 53706, AIAA Member.

${ }^{\dagger}$ Professor, Engineering Physics Department, 1500 Engineering Dr., Madison WI 53706.

Copyright 2005 by the American Institute of Aeronautics and Astronautics, Inc. All rights reserved 


\section{Nomenclature}

$\begin{array}{ll}\mathrm{A} & =\text { Ampere } \\ \mathrm{Ar} & =\text { Argon gas } \\ \mathrm{cm} & =\text { centimeter } \\ \mathrm{dia} . & =\text { diameter } \\ \mathrm{e} & =\text { electron charge } \\ \mathrm{eV} & =\text { electron volt } \\ \mathrm{J}_{\mathrm{oe}} & =\text { random electron flux } \\ \mathrm{J}_{\mathrm{oi}} & =\text { random ion flux } \\ \mathrm{mA} & =\text { milliAmpere } \\ \mathrm{m}_{\mathrm{e}} & =\text { electron mass } \\ \mathrm{m}_{\mathrm{i}} & =\text { ion mass }\end{array}$

$\begin{array}{ll}\mathrm{MHz} & =\text { megaHertz } \\ \mathrm{mTorr} & =\text { milliTorr } \\ \mathrm{n}_{\mathrm{oe}} & =\text { electron number density, cm-3 } \\ \mathrm{n}_{\mathrm{oi}} & =\text { ion number density, cm-3 } \\ \mathrm{RF} & =\text { radio frequency } \\ \mathrm{sccm} & =\text { standard cubic centimeters per minute } \\ \mathrm{T}_{\mathrm{e}} & =\text { electron temperature, eV } \\ \mathrm{V} & =\text { volts } \\ \mathrm{W} & =\text { watts of RF power } \\ \mathrm{Xe} & =\text { Xenon gas }\end{array}$

\section{Introduction}

$\mathrm{R}$ adio frequency (RF) plasmas are attractive as neutralizing sources for electric propulsion devices because they allow for an electrodeless design and provide high efficiency and long life operation. Ion and Hall thrusters use beams of positive ions for propulsion and electrons or negative ions must be introduced into the ion beam as it leaves the thruster in order to prevent the spacecraft from charging negatively and attracting the emitted positive ion beam. Traditionally, hollow cathodes have been used as neutralizing sources because of their high electron current density and relatively low power requirements. However, their operational lifetime is limited by cathode deterioration, contamination, and barium diffusion rates ${ }^{1}$ rendering them less suitable for sustained use.

Longer duration spacecraft missions that use ion propulsion, such as the Jupiter Icy Moons Mission (JIMO), will take 6-10 years for the total orbital transfer time ${ }^{2}$. While using ion propulsion for longer duration missions is very beneficial because of fuel, mass, and time savings (as opposed to impulsive chemical rocket burns), the lifetime of some operating components for ion propulsion, such as the hollow cathode, may be limited to 3-4 years ${ }^{2}$. The hollow cathode neutralizer and plasma sources that were used for the highly successful Deep Space 1 and SMART-1 missions may be limited to 3-4 years of operational lifetime due to significant erosion, sputtering, and re-deposition of material within the keeper region and surrounding areas ${ }^{2,4,5}$.

This work has developed a prototype RF plasma neutralizing device as part of NASA's HiPEP program to develop a high powered long duration ion propulsion system ${ }^{6}$. RF plasma sources provide an alternative neutralizing approach that does not consume electrode material while providing electrons, thereby allowing for a longer operational lifetime. A variety of RF sources exist including capacitive and inductive sources, which can operate without magnetic fields, and both electron cyclotron resonance (ECR) and helicon sources, which require axial magnetic fields. Helicon sources appear to be the best choice of RF plasma sources for use in ion propulsion because they can produce the highest plasma densities, up to $10^{13} \mathrm{~cm}^{-3}$ is common ${ }^{7}$, for a given RF power but they also require high magnetic field strengths and/or high RF powers. If insufficient power is available, helicon sources will operate as inductive sources. At much lower RF powers, the plasma is capacitively coupled and results in lower plasma densities.

Inductively coupled plasmas can achieve significant plasma densities, $10^{10} \mathrm{~cm}^{-3}$ to $10^{12} \mathrm{~cm}^{-3}$ and allow for a large total electron extraction current ${ }^{8}$. The current proof of principle device at the University of Wisconsin-Madison produces an inductively coupled plasma with a plasma density of $10^{10} \mathrm{~cm}^{-3}$ to $10^{12} \mathrm{~cm}^{-3} .3 .75 \mathrm{~A} / \mathrm{cm}^{2}$ of electron neutralizing current was extracted at an electron sheath (sheath where ion density is neglected) near a grounded ring located at the plasma source boundary.

This paper discusses several limiting factors in extracting a population of electrons for the purposes of neutralizing an ion thruster.

\section{Experimental Hardware}

The plasma chamber used in this experiment contains: a neutralizing cathode, made up of an ion collection cylinder, electron extraction ring, RF antenna, Faraday shield, and permanent magnets; a vacuum chamber; diagnostic tools, a Langmuir probe and an emissive probe; and a feed gas, argon. A schematic illustration of the plasma chamber containing the electrodeless plasma neutralizer and supporting vacuum hardware is shown in Fig. 1.

Neutralizing cathode. A $7.5 \mathrm{~cm}$ dia., $19 \mathrm{~cm}$ long, hollow aluminum cylinder is located within the plasma chamber as the ion collection cylinder and can be biased from 0 to $-200 \mathrm{~V}$. This cylinder is a radial boundary for the plasma and acts as a location for the formation of an ion sheath that prevents electrons from leaking to the chamber 
walls. The ion collection cylinder also has 8 axial slots $(0.5 \mathrm{~cm}$ thick), which allow the $\mathrm{dB} / \mathrm{dt}$ fields into the plasma chamber but limits the $\mathrm{dE} / \mathrm{dt}^{\text {fields }}{ }^{9}$, effectively becoming a Faraday shield. The electron extraction ring is an electrically grounded $1.25 \mathrm{~cm}$ dia. graphite ring that sits inside a boron nitride disk. This ring creates an axial boundary condition, limiting the plasma and the feed gas, and gives a potential reference for the plasma somewhere near 0 to $+2 \mathrm{~V}$. The $\mathrm{RF}$ antenna is formed from a single turn $1 / 4$ " water cooled copper pipe and operates at RF frequencies from 0.5 to $30 \mathrm{MHz}$. The permanent magnet geometry is discussed below and illustrated in Fig 2 .

Vacuum chamber. The cathode is set within a $60 \mathrm{~cm}$ long $7.5 \mathrm{~cm}$ dia. Pyrex chamber. A diffusion pump creates a base vacuum pressure of $2 * 10^{-6}$ Torr.

Diagnostic tools. A $0.75 \mathrm{~cm}^{2}$ planar graphite Langmuir probe is inserted from the right in Fig. 1 and can sweep out all axial locations in the target side of the plasma. A $0.1 \mathrm{~mm}$ dia. Tungsten emissive probe (not shown) is also inserted from the right side of the chamber and can be extended through the target side (right) of the plasma and into the source side (left), and is used to determine the plasma potential along the axis of the plasma chamber.

Feed gas. Argon feed gas is introduced into the chamber from a mass flow controller (not shown) and flows into the source region where a plasma is excited by the RF antenna.

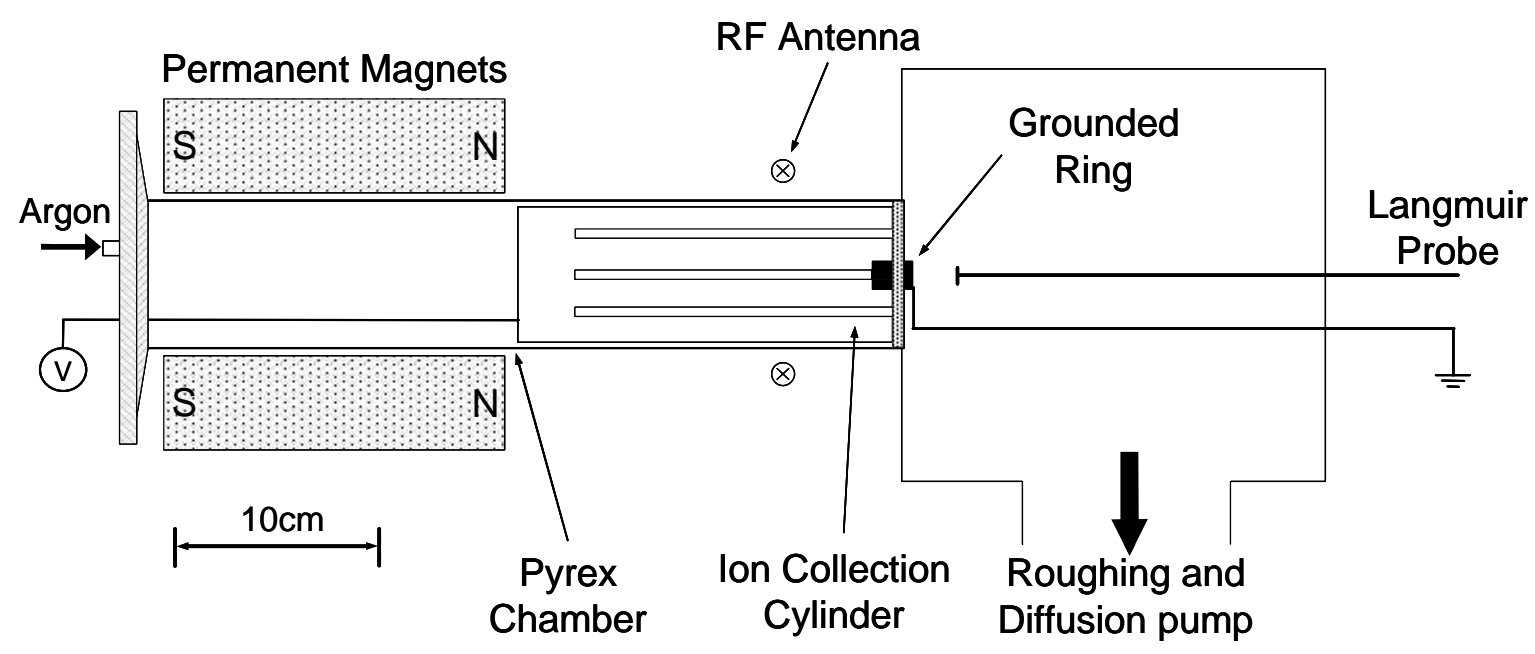

Figure 1. Schematic illustration of the plasma chamber containing the neutralizing plasma cathode with supporting vacuum hardware.

Magnetic geometry. A set of permanent magnets (Fig. 2a inset of chamber cross section), generates a solenoidal field in the axial direction of the Pyrex plasma chamber. The ferrite magnets have nearly a square cross section with exception of a cylindrical void that allows space for the Pyrex chamber. These magnets produce an expanding magnetic field in the region of the antenna and electron extraction ring. The expanding magnetic field creates a cusp, shown as point A in Fig $2 b$. Fig. $2 b$ is a plot of the equipotential magnetic field strength, created with Vizimag 3.0. This magnetic field ensures that the electrons follow the field lines that pass through the exit region of the electron extraction ring, and that fewer electrons are lost to the walls of the source region. Permanent magnets are preferred for this type of in-space neutralizer because they do not require a power source for continual operation and are relatively light weight compared to a DC power source. Fig. 2a shows a plot of the normalized magnetic field strength as a function of the axial location used to determine the magnetic geometry for the chamber, where the grounded ring experiences a magnetic field of 72 Gauss. The dashed line in Fig. $2 b$ defines where the magnetic field strength was sampled for Fig. 2a, where zero is defined by point A. 

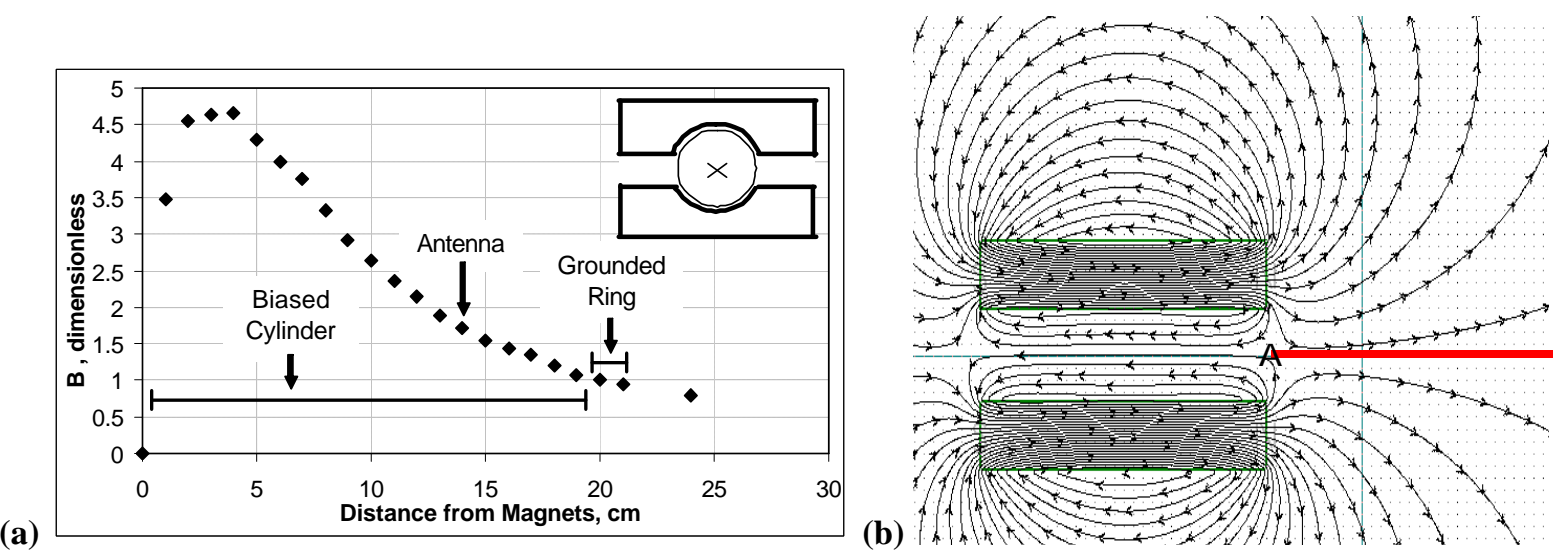

Figure 2. Normalized axial magnetic field strength from permanent magnets as a function of position. Transverse cross-section of magnets and Pyrex tube shown in inset (a), equipotential magnetic field lines for an axial cross section (b).

\section{Experimental Results and Discussion}

In order to maintain quasineutrality during steady state operation, the amount of electron loss from the source must be balanced by an equal amount of ion loss ${ }^{10}$. Because electrons and ions are born at an equal rate within the RF discharge, an efficient loss mechanism for the ions must be realized in order to extract an equal amount of electron current from the plasma source. Ion and electron losses, gas utilization, plasma density, and plasma potential effects all present limiting features on the total amount of electron current that can be extracted for neutralizing an ion thruster and are explored further in this paper.

Ion and electron losses. Electron sheaths can extract almost all of the random electron current from the system. Identifying $\mathrm{J}_{0 \mathrm{e}}$ as the random electron flux directed towards the sheath at the sheath edge

$$
\mathrm{J}_{0 \mathrm{e}}=\frac{\mathrm{n}_{0 \mathrm{e}} \mathrm{e}}{4} \sqrt{\frac{8 \mathrm{~T}_{\mathrm{e}}}{\pi \mathrm{m}_{\mathrm{e}}}}
$$

where $\mathrm{T}_{\mathrm{e}}$ is measured in $\mathrm{eV}$, and taking the ion flux $\mathrm{J}_{0 \mathrm{i}}$ equal to the Bohm current

$$
\mathrm{J}_{0 \mathrm{i}}=\mathrm{n}_{0 \mathrm{i}} \mathrm{e} \sqrt{\frac{\mathrm{T}_{\mathrm{e}}}{\mathrm{m}_{\mathrm{i}}}}
$$

the ratio of electron to ion flux associated with electrons created by ionization from Eqs. (1) and (2) is approximately equal to $\sqrt{m_{i} / m_{e}}$. The limit to the existence of an electron sheath is provided by the condition that the ion loss current to area $A_{i}$ be balanced by the electron loss current to area $A_{e}$. Assuming all the electrons are lost at the electron sheath gives

$$
A_{i} / A_{e} \approx \sqrt{m_{i} / m_{e}}
$$

assuming the electrons are confined radially. For large $A_{e}$, the electron sheath is no longer a viable solution. For sufficiently large $\mathrm{A}_{\mathrm{e}}$, only a plasma potential more positive than the grounded electrode potential, combined with an ion sheath, can provide the necessary balance of electron and ion losses.

In this experiment, ions are lost to the $7.5 \mathrm{~cm}$ dia. aluminum cylinder, with an ion loss area of $425 \mathrm{~cm}^{2}$ and the electron loss region is restricted to a small $\left(1.23 \mathrm{~cm}^{2}\right)$ aperture. An electron loss area of $1.23 \mathrm{~cm}^{2}$ implies the need of an ion loss area of at least $A_{i} \approx A_{e} \sqrt{m_{i} / m_{e}} \approx 350 \mathrm{~cm}^{2}$ for Ar and $630 \mathrm{~cm}^{2}$ for Xe.

If the source is operated at an argon plasma density of $10^{11} \mathrm{~cm}^{-3}, 1.1 \mathrm{~A}$ of electron current can be extracted through a $1.23 \mathrm{~cm}^{2}$ electron loss area if there is a $100 \%$ neutral gas utilization. If higher plasma densities are 
achieved, more current can be extracted or a correspondingly smaller electron loss area can be used, which then requires a correspondingly smaller ion loss area. The entire device is essentially area limited in that the electron extraction current can not exceed the ion extraction current that is collected by the ion loss area.

Gas utilization. For in-space neutralizer applications, it is beneficial to utilize a plasma production method that can create the largest fraction of ionization possible such that neutral gas is not wasted.

If the plasma source was $100 \%$ efficient in ionizing the neutral Ar gas as it flows through the system, and each atom is used only once as it touches the ion collection cylinder, $1 \mathrm{sccm}$ of argon is equivalent to $0.072 \mathrm{~A}$ of extraction current. The solid line in Fig. 3 is a plot of the amount of extraction current that is available from the corresponding flow rate if every atom is used 3.3 times as it passes through the plasma source and out into the target region. Fig. 3 shows that neutral $\mathrm{Ar}$ is more efficiently utilized in creating extraction current at flow rates

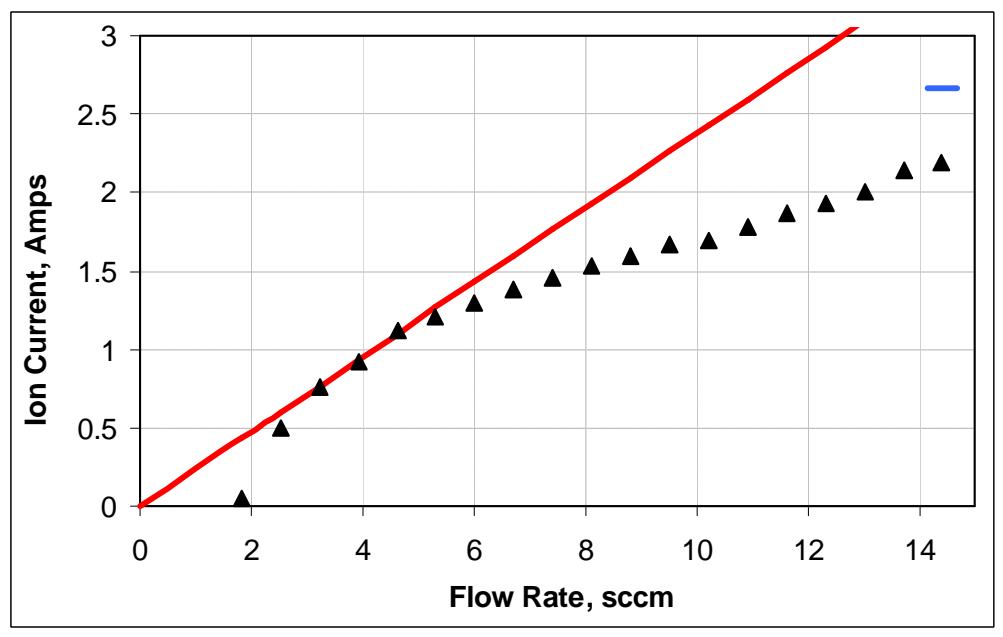

Figure 3. Current collected by ion collection cylinder (black) vs. argon flow rate, theoretical Bohm current (solid red), 3.3X flow limit (dashed), for $-40 \mathrm{~V}$ DC bias.

between $2.5 \mathrm{sccm}$ and $6 \mathrm{sccm}$. While flows rates above $6 \mathrm{sccm}$ produce larger extraction currents, the efficiency of ionization decreases until the electron current flowing through the aperture equals the Bohm current flowing to the ion collection walls (blue bar at $14 \mathrm{sccm}$ ). Here the Bohm current is found from an independent plasma density measurement within the source region. The data taken in Fig. 3 uses a fixed RF power of $340 \mathrm{~W}$ at $22 \mathrm{MHz}$. This fixed RF power is responsible for the decrease in ionization efficiency seen above $6 \mathrm{sccm}$, where the fraction of ionization decreases as the neutral flow rate is increased. The extraction current is essentially flow limited up to 6 sccm, where it then becomes plasma density limited because the RF power is fixed.

Plasma density. The amount of extractable current, Eqn. 1 and 2, is linear with plasma density which increases with RF power as seen in Fig 4. The plasma density is measured by using the ion saturation current to a small, $0.75 \mathrm{~cm}^{2}$, Langmuir probe positioned axially in the target side of the chamber. Fig. 4 is a plot of the amount of ion current, and hence an equal amount of electron current, that is extracted from the plasma source as the RF power is varied from $60 \mathrm{~W}$ to $340 \mathrm{~W}$ at a frequency of $22 \mathrm{MHz}$ and Ar flow rate of $14 \mathrm{sccm}$. The ion collection cylinder was kept at $-40 \mathrm{~V}$ (white) and $-80 \mathrm{~V}$ (black) during the power scan. The extracted current increases linearly with RF power and does not indicate a saturation point at high RF powers, indicating room for future progress. At RF powers between $60 \mathrm{~W}$ and

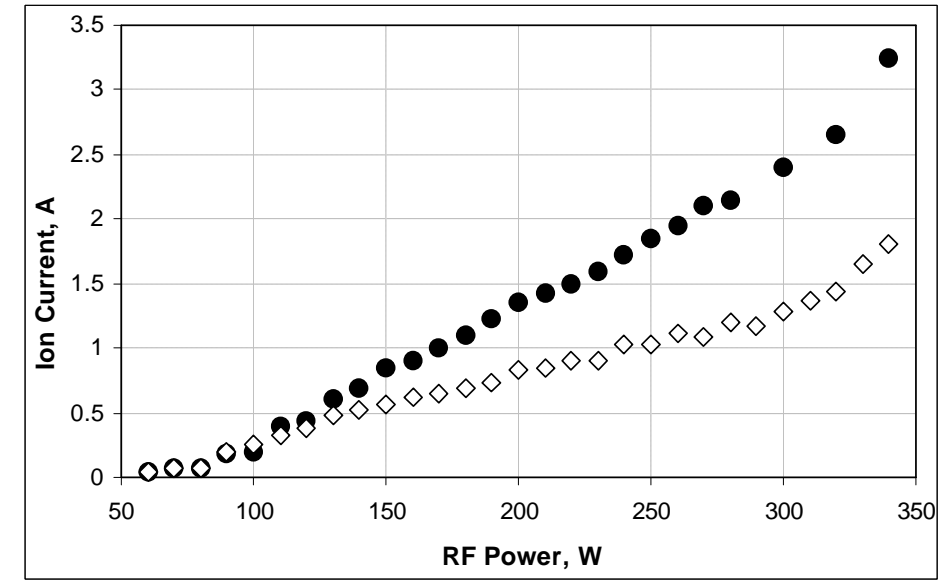

Figure 4. Current collected by ion collection cylinder as a function of RF power. DC bias on the ion cylinder of $-40 \mathrm{~V}$ (white) and -80V (black), $14 \mathrm{sccm}$ Argon.

90W the plasma does not visually fill the entire ion collection cylinder, thus decreasing the effective ion loss area, which explains the decreased collection current. The RF power supply used in this experiment is limited to $340 \mathrm{~W}$, however further increases in RF power would increase the plasma density and ionization efficiency at higher flow rates, thereby increasing the limiting Bohm current and total extraction current.

Fig. 5 shows an increase in the amount of current that can be extracted from the plasma, here as ion current to the ion collection cylinder (black diamonds), and electron current to a Langmuir probe (black circles) as a function of the DC bias on the ion collection cylinder. As the voltage is decreased from $0 \mathrm{~V}$ to $-60 \mathrm{~V}$, the ion collection 
cylinder repels a larger number of electrons away from the walls. This increases the local plasma density, which then allows the ion collection cylinder to collect more extraction current. Because the Langmuir probe takes up $60 \%$ of the area of the exiting electron stream, a normalization is performed, white circles in fig. 5 , in order to estimate the total amount of extracted electron current through the grounded ring. The normalized electron current agrees closely with the total amount of ion extraction current and shows that all of the electrons that are lost within the system are lost through the electron extraction orifice.

Plasma potential. One complication to the understanding of current extraction from the plasma source is the plasma potential difference between the source side and the target side. Regardless of the bias on the ion collection cylinder in the source side, the

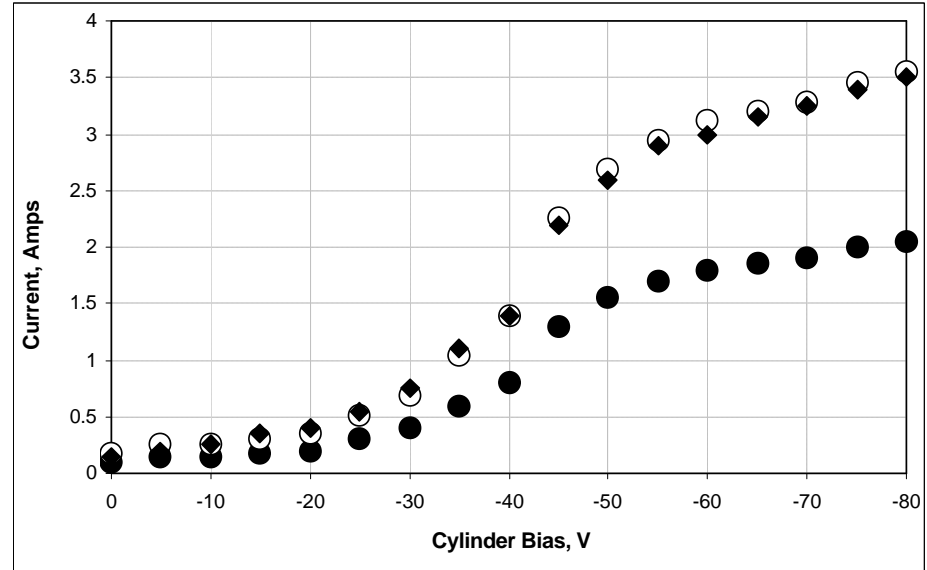

Figure 5. Ion current collected by the ion collection cylinder (diamond), extracted electron current collected by a Langmuir probe (black circle), normalized electron current collected (white circle) as a function of DC bias on the ion collection cylinder. Using 340W RF power, 14 sccm Argon. plasma potential in the target side remained above that of the plasma source region. The plasma potential within the source region remained more positive than the ion collection cylinder, $-25 \mathrm{~V}$ compared to $-80 \mathrm{~V}$ respectively, thereby giving rise to ion loss through an ion sheath at the ion collection cylinder within the source region. Fig 6 . shows that the plasma potential in the target region (dashed line) remains more positive than the plasma potential in the source region (solid line), indicating the existence of an electron sheath that is extracting electrons. Inside of the grounded ring, the plasma potential is held within $+/-1 \mathrm{~V}$ of ground potential, previously demonstrated by Severn and Hershkowitz $^{11}$.

The plasma remains more positive than the ion collection cylinder, but does saturate at $-25 \mathrm{~V}$ as seen in Fig. 6 . The target (dashed) region of the chamber remained at a higher potential compared to the plasma source side, where electrons are not lost to the ion collection cylinder because the difference in potential between the source plasma and the ion collection cylinder has $e \Delta \phi / T_{e}>>1$. The maximum electron current to the grounded ring never exceeds $10 \mathrm{~mA}$. The significant amount of ion current that is extracted at the ion collection cylinder indicates that there is a considerable non-ambipolar electron loss in the system.

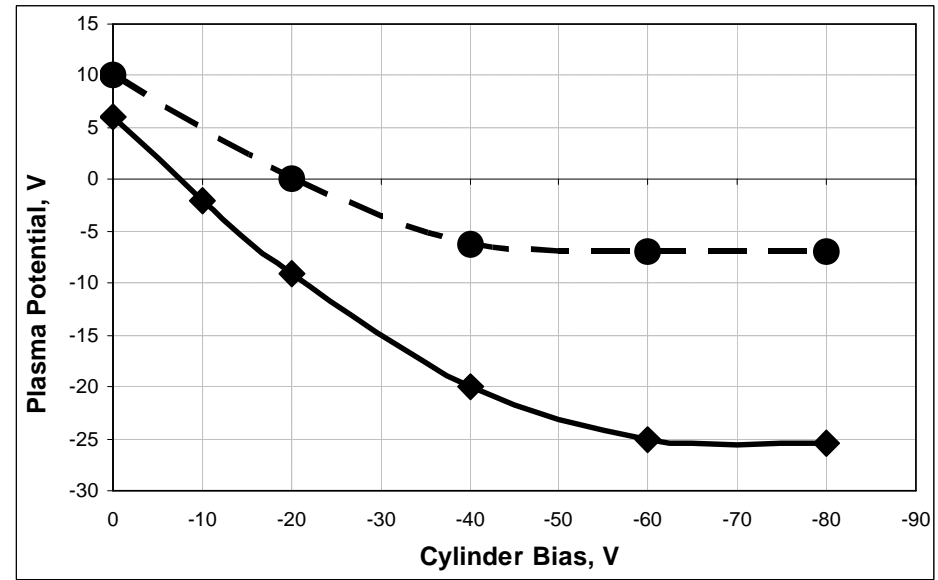

Figure 6. Plasma potential in the source (solid) region and the target (dashed) region as a function of the DC bias applied to the ion collection cylinder for 14 sccm Argon.
The ion collection cylinder acts as a Faraday shield, composed of a $19 \mathrm{~cm}$ long cylinder of aluminum with 8 axial slits. By using the ion collection cylinder as a Faraday shield the plasma potential did not fluctuate, but remained at a fixed value. If the ion collection cylinder / Faraday shield is slid to the left (in Fig. 1) so that it is no longer underneath the antenna, a large $\mathrm{AC}$ fluctuating plasma potential is created, as seen with emissive probe data. Without the use of a Faraday shield, there is significant capacitive coupling and the plasma potential oscillates back and forth with a peak to peak value of over $100 \mathrm{~V}$. The cancellation of the fluctuating plasma potential, by using a Faraday shield, is beneficial to the RF plasma neutralizer because it allows for larger and more stable extraction currents. 


\section{Conclusion}

Inductively coupled or helicon RF plasma sources appear to be the best choice for use as a neutralizing plasma source for ion thrusters due to the large ionization fraction and high plasma densities they produce. Helicon sources can ultimately generate higher plasma densities but inductively coupled plasmas produce a sufficient plasma density for many or all of the neutralizing needs of ion thrusters without requiring large magnetic fields.

An electron sheath as used in this experiment allows the extraction of all or most of the random electron flux through an orifice that is proportionally smaller than the ion loss area located within the plasma source. In addition, this prototype device has given evidence of a substantial electron neutralizing current, with a maximum of $3.75 \mathrm{~A}$. This was achieved while using $14 \mathrm{sccm} \mathrm{Ar}, 340 \mathrm{~W}$ of RF power at $22 \mathrm{MHz}$, and $-80 \mathrm{~V}$ DC bias on the ion collection cylinder. The ion collection cylinder, with an area of $425 \mathrm{~cm}^{2}$, provided the necessary ion loss area, while a smaller grounded ring was used to extract the electrons through an electron sheath into the target region of the plasma chamber. Unique to this proof of principle neutralizing RF plasma device is the possibility of scaling the neutralizing electron current based on the total amount of ion collection area within the source plasma. This electron current scaling can be done independently of other system parameters such as gas flow rate, RF power, and DC bias of the ion collection cylinder. The total amount of neutralizing extraction current of this device is ultimately limited by the ion loss area, neutral gas flow rate, and plasma density.

Inductive sources thus appear to be sufficient for neutralizing in-space ion thrusters, where larger electron extraction currents can be produced by larger RF powers, larger neutral gas flow rates, larger ion loss areas and correspondingly larger electron loss areas. This inductively coupled plasma could thus be useful as an electron source for long duration space exploration missions using ion propulsion.

\section{Acknowledgments}

The research described in this paper was carried out by the University of Wisconsin - Madison, under a contract with the National Aeronautics and Space Administration in support of the High Power Electric Propulsion project.

\section{References}

${ }^{1}$ Goebel, D. M., et al., "Extending Hollow Cathode Life for Electric Propulsion in Long-Term Missions," AIAA-2004-5911, space 2004 conference and exhibit, 28 - 30 September 2004, San Diego, California

${ }^{2}$ Oleson, S., "Jupiter Icy Moons Orbiter: Challenges for Electric Propulsion," 40th AIAA-2004-3449, AIAA/ASME/SAE/ASEE Joint Propulsion Conference \& Exhibit 11- 14 July 2004, Fort Lauderdale, FL

${ }^{3}$ Sarver-Verhey, T., “28,000 Hour Xenon Hollow Cathode Life Test,” IEPC Paper 97-168, November 1997.

${ }^{4}$ Sengupta, A., Brophy, J. R., Goodfellow, K. D. "Status of the Extended Life Test of the Deep Space 1 Flight Spare Ion Engine After 30,352 Hours of Operation,” AIAA 03-4558, July 2003.

${ }^{5}$ Sarver-Verhey, T., "Scenario For Hollow Cathode End-Of-Life," 26th International Electric Propulsion Conference AIAA, Kitakyushu, Japan, October 17-21, 1999.

${ }^{6}$ Elliott, F. W., Foster, J. E., and Patterson, M. J., "An Overview of the High Power Electric Propulsion (HiPEP) Project," AIAA-2004-3453, 40th AIAA/ASME/SAE/ASEE Joint Propulsion Conference \& Exhibit 11- 14 July 2004, Fort Lauderdale, FL

${ }^{7}$ Gilland, J., Breun, R., Hershkowitz, N., "Neutral pumping in a helicon discharge," Plasma Sources, Science and Technology, v 7, n 3, Aug. 1998, p 416-22.

${ }^{8}$ Hershkowitz, N., “Sheaths: More Complicated Than You Think,” Phys. Plasmas 055502, 2005

${ }^{9}$ Godyak, R. B. Piejak, and B. M. Alexandrovich, Plasma Sources Sci. Technol. 4, $332 \sim 1995$.

${ }^{10}$ F Chen, "Physics of Helicon Discharges," AIP review articles, 1996, page 10

${ }^{11}$ Severn, Hershkowitz, N., "Radial Control Of The Electrostatic Potential In A Tandem Mirror With QUADRUPOLE End Cells,” Physics Of Fluids B-Plasma Physics 4 (10): 3210-3215 OCT 1992 